\title{
Heterogeneous Specular and Diffuse 3-D Surface Approximation for Face Recognition Across Pose
}

\author{
Xiaozheng Zhang and Yongsheng Gao, Senior Member, IEEE
}

\begin{abstract}
This paper proposes a novel heterogeneous specular and diffuse (HSD) 3-D surface approximation which considers spatial variability of specular and diffuse reflections in face modelling and recognition. Traditional 3-D face modelling and recognition methods constrain human faces with either the Lambertian assumption or the homogeneity assumption, resulting in suboptimal shape and texture models. The proposed HSD approach allows both specular and diffuse reflectance coefficients to vary spatially to better accommodate surface properties of real human faces. From a small number of face images of a person under different lighting conditions, 3-D shape and surface reflectivity property are estimated using a localized stochastic optimization method. The resultant personalized 3-D face model is used to render novel gallery views under different poses for recognition across pose. The proposed approach is evaluated on both synthetic and real face datasets and benchmarked against the state-of-the-art approaches. Experimental results demonstrated that it can achieve a higher level of performances in modelling accuracy, algorithm reliability, and recognition accuracy, which suggests that face modelling and recognition beyond the Lambertian and homogeneity assumptions is a feasible and better solution towards pose-invariant face recognition.
\end{abstract}

Index Terms-3-D modelling, face approximation, face recognition, heterogeneity, pose variation, reflectivity.

\section{INTRODUCTION}

$\mathbf{F}$ ACE images under pose variations are largely associated with both surface reflectance property and 3-D structure of the face. Most of the existing face recognition techniques [11], [15], [23], [47], [55] approximate face surfaces under the Lambertian assumption which neglects specular reflection. In reality, however, human faces reflect light both diffusely and specularly [8]. Without specular reflection, face modelling under the Lambertian assumption results in not only suboptimal surface reflectivity maps, but also distorted 3-D shapes. Any of the two errors can adversely affect the performance of the subsequent face recognition. To address this problem, the Phong model

Manuscript received April 07, 2011; revised September 18, 2011; accepted September 21, 2011. Date of publication September 29, 2011; date of current version March 08, 2012. This work was supported by the Australian Research Council (ARC) under Discovery Grant DP0451091. The associate editor coordinating the review of this manuscript and approving it for publication was Dr. Jaihie Kim.

$\mathrm{X}$. Zhang is with the Queensland Research Laboratory, National ICT Australia and Institute for Integrated and Intelligent Systems, Griffith University, Nathan, Qld 4111, Australia (e-mail: paul.zhang@nicta.com.au, x.zhang@griffith.edu.au).

Y. Gao is with the Griffith School of Engineering, Griffith University, Nathan, Qld 4111, Australia, and Queensland Research Laboratory, National ICT, Australia (e-mail: yongsheng.gao@griffith.edu.au,yongsheng.gao@nicta.com.au).

Digital Object Identifier 10.1109/TIFS.2011.2170068 with a homogeneity assumption on specular reflection components has been used in [6] for face modelling and recognition, which achieved promising performance improvements. However, human face surfaces are not homogeneous where diffuse and specular reflectivities are both spatially variable. This paper proposes a novel heterogeneous specular and diffuse (HSD) 3-D surface approximation approach which considers spatial variability of specular and diffuse reflections for better 3-D face modelling and face recognition across various poses.

Recently, 3-D model-based techniques have become the most successful face recognition approaches, especially when invariance to pose and illumination variations is desired [32]. Based on the pixel intensities of face images, the illumination cone model [11] and 3-D morphable model [6] have been successfully applied to describe human faces from multiple images and from a single image with prior knowledge of human faces, respectively. However, both methods either neglect the specular reflection component or constrain the reflectance map to be homogeneous. Due to the complexity of human face surfaces, these restrictions are oversimplistic to faithfully describe human faces in reality and affect the accuracy of face modelling and recognition. It is desirable to model human faces both beyond the Lambertian assumption to cover specular reflection and to be heterogeneous to preserve identity-related facial details [32]. This paper proposes a novel HSD 3-D surface approximation approach, which generalizes the image-based 3-D face modelling for face recognition across poses. Personalized 3-D face models are estimated from a small number of face images under different lighting conditions with a fixed viewpoint. Both shape and surface reflectance properties are calculated locally through minimization of the image differences between the original images and their estimations. Stochastic computational methods and integrability enforcement are employed to handle the nonlinearity and inconsistency issues in the shape and reflectance parameter optimization processes to achieve valid results. In experiments, modelling accuracies and face recognition performance of the proposed HSD approach have been evaluated on synthetic data and real face image database. Compared to the diffuse-only model and the homogeneous specular reflectance model, the proposed HSD approach was proved more accurate in 3-D shape reconstruction through better approximation of real surface properties. On the publicly available CMU pose-illumination-expression (PIE) database, the proposed HSD approach achieved higher recognition rates than the state-of-the-art face recognition techniques in handling pose variations.

The remainder of the paper is organized as follows. Section II provides a background for the proposed research which in- 
cludes topics on face recognition, face modelling, human face skins, and reflectance models. In Section III, we elaborate on the proposed HSD approach. The optimization details, the preprocessing of lighting estimation, and the postprocessing for perturbation alleviation are also included in this section. Experimental results on both synthetic and publicly available face database are given in Section IV. Finally, the paper concludes in Section V.

\section{RELATED WORKS}

Recently, pose variations in face recognition captured growing interest from researchers in the fields of computer vision and pattern recognition [52]. An effective strategy to handle pose variations is to use the assistance of 3-D face models, because human heads are nonplanar 3-D objects so that viewpoint changes take place in the 3-D space. Three-dimensional model-based face recognition using personalized 3-D models estimated from 2-D images [6], [11], [20], [46], [49]-[51] has shown its promise. Such face recognition algorithms perform on a recognition-by-synthesis mechanism in the 2-D image spaces or in dimension reduction over shape and surface reflective parameters in the 3-D space. Consequently, the performances of these techniques are greatly dependent on the accuracy of the estimated 3-D models.

Image-based face modelling considers facial textures (i.e., pixel intensities) as critical clues and estimates 3-D face shapes and surface reflectance properties by reversing image formation processes. Yuille et al. [46] extended photometric stereo methods [17], [43] and made the first attempt on image-based face modelling. Georghiades et al. [11] proposed a linear framework for image-based face modelling, which greatly improved the tractability of the complicated process. Using spherical harmonics of incident lights, Ramamoorthi [30], and Basri and Jacobs [3] generalized the low-dimensional linear subspace theory for representing the images of convex Lambertian objects from the conditions of single directional light sources to cases of multiple extended light sources. Zhou et al. [55] approximated image variations of different human faces as a single linear subspace under Lambertian assumption so that single gallery per person was made possible. Zhang and Samaras [47] further extended Zhou's method [55] by incorporating the theory of spherical harmonics illumination representation to handle multiple general light sources. In the cost of the tractable modelling framework, linear processes have to reside on certain oversimplistic assumptions. Other researchers chose to model human faces more faithfully and in nonlinear spaces. Blanz and Vetter [5], [6] proposed a 3-D morphable model based on prior knowledge of human faces and presented a successful approach on illumination-and pose-invariant face recognition through 3-D modelling. Jiang et al. [20] estimated face shapes purely based on facial features and principal components of the prior knowledge of 3-D faces and built a real-time face recognition system. Romdhani and Vetter [31] incorporated additional image clues such as edges and specular highlights into the process of imagebased 3-D modelling to alleviate the instability of the model fitting process in [5] and [6].

In reflection theory, image-based modelling techniques apply bidirectional reflectance distribution function (BRDF) to relate

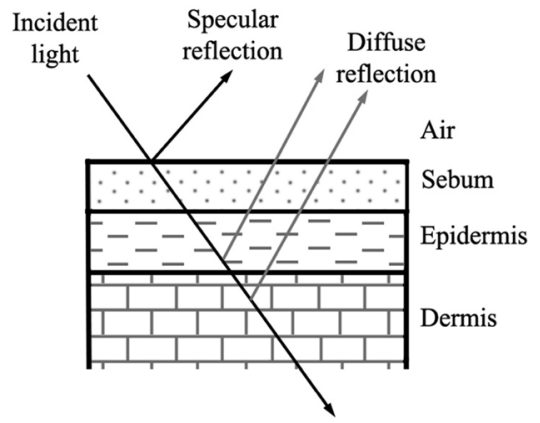

Fig. 1. Incident light and reflections in a three-layer skin model.

the object geometry information (in the form of normal directions) to the image intensities (in the form of reflected light intensities). Common analytic reflectance models used in 3-D face modelling and recognition are the Lambertian model [3], [11], [30], [46], [55] and Phong model [5], [6]. Because the Lambertian model only considers diffuse reflection and neglects specular reflection, its description is only feasible for dull matte materials such as chalks. The Phong model, on the other hand, considers both diffuse and specular reflection in a simple analytical form. Optical studies usually model human skin as three layers: sebum layer (mixture of sebum, lipids, and sweat), epidermis layer (stratum corneum and malpighian), and dermis layer (papillary dermis and reticular dermis) [24], [25], [29], as shown in Fig. 1. In an infinitesimal surface area, these three layers are approximately parallel [41]. When the face skin is illuminated, specular reflection occurs mainly on the smooth air-sebum interface and is mainly dependent on the thickness and characteristics of the sebum layer. Sebum production varies with age, gender, and race. Statistically, young, male, or black skin produces more sebum than aged, female, or white skin [25]. The presence of sebum layer in face skin explains histologically why human faces exhibit specular reflection and cannot be modelled purely as Lambertian surfaces. The rest of the incident light penetrates these layers and is scattered to diffuse lights with equal intensity in all directions. Diffuse reflection then occurs on epidermis or dermis whose reflectivity is mainly dependent on the amount of melanin pigment, the amount of blood-borne hemoglobin pigments, and the thickness of collagen [29]. Both of the thickness of the sebum layer and the amount of melanin pigments vary spatially along human face surfaces, which make both diffuse and specular reflection components heterogeneous as stated in [8]. Therefore, specular and diffuse reflectivities are both histological characteristics different from person to person and should be approximated as such in face modelling and recognition processes. None of the above techniques, however, allows both specular and diffuse reflectance maps to vary spatially along face surfaces, which could be oversimplistic to reality. Based on this histological analysis of human skin and related reflections, this paper attempts to model 3-D face shapes and surface reflectance properties by assuming face surfaces as heterogeneous specular and diffuse reflecting surfaces to better approximate reflectivities of real face skin.

People are incredibly sensitive to the subtle differences of face appearances [42] and these shape and face skin representations are also critical classification features for most face recog- 
nition algorithms, no matter whether they are 2-D image-based or 3-D model-based. Slight deviations on the estimated 3-D face model often result in unappealing images in face rendering and classification errors in face recognition. To overcome the inaccuracy in modelling face surfaces as Lambertian surfaces, limited research has been attempted by treating face surfaces as reflective surfaces beyond Lambertian assumption. Blanz and Vetter [6] modelled the 3-D face based on the Phong model [28], which can be expressed as

$$
I_{\mathrm{PH}}=I_{a} k_{d}+I_{i} k_{d} \cos \beta_{l}+I_{i} k_{s} \cos ^{n_{s}} \beta_{r}
$$

where $I_{\mathrm{PH}}$ is the image intensity in the Phong model under an ambient light $I_{a}$ and a directional light $I_{i}, n_{s}$ is the specular reflection exponent, and $\beta_{r}$ is the angle between reflecting direction and viewing direction. The surface properties of specular reflection of human faces in the above method, i.e., $k_{s}$ and $n_{s}$ in [6], were assumed to be homogeneous. This approximation on specular reflection holds when surfaces exhibit homogeneity or little variance on specular reflectivity, which in most cases are man-made objects such as a lacquered globe. Most natural objects including human faces, however, exhibit inhomogeneous (i.e., heterogeneous) specular reflectivity [8], [42]. The fixed specular reflectance coefficients throughout the modelling process on such objects will limit the variability of both shape and texture parameters moving toward better face models. The modelling approach that considers both diffuse and specular reflections spatially variable is therefore desirable. The generalization of face surface assumptions in this research better approximates the real surface properties of human faces, which in result are beneficial to shape and reflectance modelling.

\section{Heterogeneous Specular and Diffuse 3-D SURFACE APPROXIMATION}

Under certain surface reflection assumptions, image-based 3 -D modelling typically uses pixel intensities from 2-D images as the cue for model estimation. Consequently, the surface reflection assumptions play a critical role in the modelling process, which affect both estimated shape model and surface reflectance maps. In Section III-A, the specific surface reflection assumptions are elaborated and examined in the context of 3-D surface approximation and modelling.

\section{A. Reflectance Models and Image-Based 3-D Modelling}

Generally, the reflection from an object's surface can be described as a linear combination of diffuse reflection and specular reflection [35]. For face surfaces, diffuse reflection takes place in epidermis and dermis layers of human skin while specular reflection occurs in the sebum layer as discussed in Section II. The reflected intensity in an image is expressed as

$$
I_{r}=k_{d} \rho_{d} I_{i}(\boldsymbol{n} \cdot \boldsymbol{l})+k_{s} \rho_{s} I_{i}(\boldsymbol{n} \cdot \boldsymbol{l})
$$

where $\boldsymbol{n}$ is the unit normal vector, and $\boldsymbol{l}$ is the unit direction of the incident light, $\rho_{d}$ and $\rho_{s}$ are the diffuse and specular bidirectional reflectivities [26], respectively, which are usually associated to surface geometry and properties, lighting and viewing conditions. $k_{d}$ and $k_{s}$ are diffuse and specular reflectance coefficients, respectively, which are intrinsic reflection properties of the object's surface. Although the reflection mechanism in reality is very complicated, many reflectance models provide reasonable approximations at different levels of accuracy and complexity, such as Lambertian model [22], Phong model [28], He model [18], etc. The diffuse component of the reflection in (2) can be well approximated using Lambertian model in which $\rho_{d}$ is assumed to be 1 [39]. If the surface is dull matte (e.g., chalks), it is called a Lambertian surface which only exhibits diffuse reflection. Under such circumstances, the reflected intensity has a simple form as

$$
I_{r L}=k_{d} I_{i}(\boldsymbol{n} \cdot \boldsymbol{l}) .
$$

Due to its simplicity, the Lambertian model is widely used in 3-D face modelling and/or recognition. When applied to surfaces exhibiting specular reflection (e.g., human faces), however, the modelling results are suboptimal because it neglects specular reflection.

To account for both specular and diffuse reflections, many reflectance models add one or more specular reflection components on the Lambertian reflection component. For instance, the Phong model [28] describes the reflected intensity as

$$
I_{r P}=k_{d} I_{i}(\boldsymbol{n} \cdot \boldsymbol{l})+k_{s} I_{i}(\boldsymbol{r} \cdot \boldsymbol{v})^{n_{s}}
$$

where $\boldsymbol{r}=2(\boldsymbol{n} \cdot \boldsymbol{l}) \boldsymbol{n}-\boldsymbol{l}$ is the reflecting direction and $\boldsymbol{v}$ is the viewing direction. Although the Phong model is an empirical model with little physical basis, it has been widely used due to its simplicity. Torrance and Sparrow [40] modelled the diffuse and specular reflections based on applied optics as

$$
I_{r T}=k_{d} I_{i}(\boldsymbol{n} \cdot \boldsymbol{l})+k_{s} I_{i} F G \frac{e^{-\beta_{h}^{2} / m_{s}^{2}}}{(\boldsymbol{n} \cdot \boldsymbol{v})}
$$

where $F$ is the Fresnel equation specifying how the light is reflected from each smooth microfacet, $G$ is the geometrical attenuation factor taking into account inter-microfacet shadowing, and the halfway angle is expressed as $\beta_{h}=\arccos (\boldsymbol{l} \cdot \boldsymbol{h})(\boldsymbol{h}$ is the halfway vector between the light direction and the reflecting direction). The comparison between the Phong model and Torrance-Sparrow model reveals that the two models produced similar results when the incident angle was small. Only when the incident angle is large $\left(\sim 70^{\circ}\right)$ were their reflections are noticeably different, which indicates the Phong model is a fairly good approximation of reflectance models with physical basis [7].

In addition, when the object is in a bright environment, an ambient term is usually included in the reflectance model as

$$
I_{r a}=k_{a} I_{a}
$$

where $I_{a}$ is ambient light which is assumed to be uniform and directionless [9], and $k_{a}$ is the ambient reflectance coefficient.

Given a 2-D face image, the image intensity of a point on the surface can be estimated using (4) or (5) and is denoted as $\hat{I}$. The estimated intensity should resemble the real input image $I$ of the 
same point. Ideally, all shape, reflectance, and environmental coefficients (surface normal $\boldsymbol{n}$, reflectance coefficients $k_{a}, k_{d}$, $k_{s}$, lighting conditions $I_{a}, I_{i}, \boldsymbol{l}$, and viewing direction $\boldsymbol{v}$ ) can be estimated inversely by minimizing the difference of $\hat{I}$ and $I$. However, it is impossible to separate shape, reflectance, and environmental coefficients from a single 2-D face image without constraints or prior knowledge. To make this inverse estimation tractable, multiple 2-D images are usually used. One of the successful 3-D modelling approaches, photometric stereo [11], [55], uses a set of images with fixed viewpoint under variable illuminations to inversely model 3-D objects. Another strategy of analyzing object geometry from a single illuminated image is shape from shading [48]. Typical photometric stereo and shape from shading techniques assume known [38] or uniform [19], [37], [44] reflectance of the object surface to extract shape information, but more recent work began to extend their functionality to include surfaces with unknown heterogeneous reflectance maps. Based on the Lambertian model, several algorithms successfully operated under the conditions of unknown single lights [11], [46], unknown general illuminations [4], and from a mixture of images of different objects [47], [55]. To deal with the limitation of the Lambertian assumption incapable of handling specularity, specularity is detected using a fine-tuned illumination combination and the images exhibiting specularity are then excluded from the training set [2], [45], [53], assuming that for each pixel there exist a number of images on which the reflections are merely diffuse (Lambertian). The existing methods for analyzing human face surface reflectance properties are either based on the Lambertian assumption considering only diffuse reflection from the face surface [11], [15], [46] or based on the homogeneous assumption considering that the material has a uniform distribution on certain reflectance coefficients [6]. The image-based 3-D modelling that considers both diffuse and specular reflections spatially variable is challenging and remains an open question. In our proposed HSD 3-D surface approximation approach, these limitations are lifted, i.e., we do not require pure Lambertian reflection for certain pixels and we simultaneously optimize both specular and diffuse reflectance coefficients in order to estimate an optimal shape and surface model.

\section{B. Surface Approximation and Modelling}

Suppose $t$ images $\boldsymbol{I}_{1}, \boldsymbol{I}_{2}, \ldots, \boldsymbol{I}_{t}$ of a human face under $t$ different lighting conditions from a viewing direction $\boldsymbol{v}=(0,0,1)^{T}$ are given. For the $j$ th image, the face is illuminated by ambient light with ambient intensity $I_{a j}$ and a distant light with an incident intensity $I_{i j}$ from direction $\boldsymbol{l}_{j}$ defined as

$$
\boldsymbol{l}_{j}=\left(\sin \theta_{j} \cos \phi_{j}, \sin \phi_{j}, \cos \theta_{j} \cos \phi_{j}\right)^{T}
$$

where $\theta_{j}$ and $\phi_{j}$ are azimuth and zenith incident angles, respectively. The lighting conditions can be represented as four $s$-dimensional vectors $\boldsymbol{S}_{a}=\left(s_{a 1}, s_{a 2}, \ldots, s_{a t}\right)^{T}$, $\boldsymbol{S}_{i}=\left(s_{i 1}, s_{i 2}, \ldots, s_{i t}\right)^{T}, \boldsymbol{\theta}=\left(\theta_{1}, \theta_{2}, \ldots, \theta_{t}\right)^{T}$, and $\boldsymbol{\phi}=\left(\phi_{1}, \phi_{2}, \ldots, \phi_{t}\right)^{T}$. Surface normal $\boldsymbol{n}$ of point $(x, y)$ is calculated as

$$
\boldsymbol{n}=\frac{\left(-z_{x},-z_{y}, 1\right)^{T}}{\sqrt{z_{x}^{2}+z_{y}^{2}+1}} .
$$

The specular reflectivity for point $(x, y)$ is denoted as $\boldsymbol{\rho}_{s}(x, y)=\left(\rho_{s 1}, \rho_{s 2}, \ldots, \rho_{s t}\right)^{T}$. The shape and surface reflective information can then be estimated by minimizing the sum square differences at $(x, y)$ between $\boldsymbol{I}_{j}$ and $\hat{\boldsymbol{I}}_{j}$, which is written as

$$
E=\sum_{j=1}^{t}\left\{I_{j}-I_{a j} k_{a}-I_{i j}(\boldsymbol{n} \cdot \boldsymbol{l})\left[k_{d}+k_{s} \rho_{s j}\right]\right\}^{2} .
$$

The specular reflectivity $\rho_{s}$ is a variable associated with many environmental and intrinsic parameters such as normal directions, surface reflectance coefficients, lighting conditions, viewing directions, etc. The aforementioned reflectance models are to approximate the specular reflectivity using explicit expressions of those parameters. After selecting a specific reflectance model, the inverse estimation of shape and surface properties is then practical. In this work, the Phong model and Torrance-Sparrow model are used and investigated. Other reflectance models could also be fit into the proposed framework with further investigations of computational feasibility and tractability.

When using the Phong model, (4) is integrated into (9) as

$$
E_{P}(x, y)=\sum_{j=1}^{t}\left\{I_{j}-I_{a j} k_{a}-I_{i j}\left[\begin{array}{c}
k_{d}\left(\boldsymbol{n} \cdot \boldsymbol{l}_{j}\right) \\
+k_{s}\left(\boldsymbol{r}_{j} \cdot \boldsymbol{v}\right)^{n_{s}}
\end{array}\right]\right\}^{2} .
$$

For the Torrance-Sparrow model, we assume that the Fresnel equation $(F)$ is constant and geometric attenuation factor $(G)$ is 1 as done in [13] and [16]. The objective function based on the Torrance-Sparrow model is then expressed as

$$
\begin{aligned}
E_{T}(x, y)=\sum_{j=1}^{t} & \left\{I_{j}-I_{a j} k_{a}\right. \\
& \left.-I_{i j}\left(\boldsymbol{n} \cdot \boldsymbol{l}_{j}\right)\left[k_{d}+\frac{k_{s}}{(\boldsymbol{n} \cdot \boldsymbol{v})} e^{-\beta_{h j}^{2} / m_{s}^{2}}\right]\right\}^{2}
\end{aligned}
$$

where $\beta_{h j}=\arccos \left(\boldsymbol{n} \cdot \boldsymbol{h}_{j}\right)$. Although the complete 3-D modelling requires inverse estimation of all lighting conditions, shape and reflectance parameters from 2-D images, the lighting estimation is relatively independent of shape and reflectance estimation since lighting parameters are globally consistent parameters for the whole scene while the shape and reflectance parameters are spatially varying. In this work, the lighting estimation is performed as the preprocessing step before shape and reflectance estimation, if the lighting conditions of the images are unknown. The lighting estimation used in our experiments will be introduced in Section III-C, but other existing algorithms (e.g., [33], [34], and [54]) can also be used. In (10) or (11), the 
shape and reflectance parameters to be estimated are $\boldsymbol{n}, k_{a}, k_{d}$, $k_{s}$ and $n_{s}\left(\right.$ or $\left.m_{s}\right)$, where normal direction $\boldsymbol{n}$ is expressed using azimuth angle $\theta_{n}$ and zenith angle $\phi_{n}$ as

$$
\boldsymbol{n}=\left(\sin \theta_{n} \cos \theta_{n}, \sin \phi_{n}, \cos \theta_{n} \cos \phi_{n}\right)^{T}
$$

Thus, each optimization has six parameters, i.e., $\theta_{n}, \phi_{n}, k_{a}, k_{d}$, $k_{s}$, and $n_{s}$ (or $\left.m_{s}\right)$.

To take the attached shadows into consideration, the dot products in (10) or (11) are all semi-positive. Cast shadows exist in the region where the incident lights are blocked by other parts of the human face. To handle the effects of cast shadows, prior knowledge of human faces is used in the optimization process. Provided a generic shape of human faces and lighting directions, the cast shadows are calculated using z-buffering [9]. In the objective function, if a point under a certain incident light is in cast shadows, the diffuse and specular reflections are both set to zero and the difference between the estimated and the original intensities becomes the difference between the ambient reflection and the original intensity. The objective functions [(10) and (11)] can be rewritten as

$$
E_{P}(x, y)=\sum_{j} E_{P j}(x, y)^{2}
$$

and

$$
E_{T}(x, y)=\sum_{j} E_{T j}(x, y)^{2}
$$

where

$$
\begin{aligned}
& E_{P j}(x, y)= \\
& \begin{cases}I_{j}-I_{a j} k_{a}-I_{i j}\left[k_{d}\left(n \cdot l_{j}\right)+k_{s}\left(r_{j} \cdot v\right)^{n_{s}}\right], & \text { not in shadow } \\
I_{j}-I_{a j} k_{a}, & \text { in shadow }\end{cases}
\end{aligned}
$$

and

$$
\begin{aligned}
& E_{T j}(x, y)= \\
& \begin{cases}I_{j}-I_{a j} k_{a}-I_{i j}\left(n \cdot l_{j}\right)\left[k_{d}+\frac{k_{s}}{(n \cdot v)} e^{-\beta_{h j}^{2} / m_{s}^{2}}\right], & \text { not in shadow } \\
I_{j}-I_{a j} k_{a}, & \text { in shadow }\end{cases}
\end{aligned}
$$

Both objective functions are nonlinear, which may contain multiple local minima. Stochastic optimization methods can be utilized to find the desired global minimum avoiding of being trapped in the local minima. In this work, a genetic algorithm is employed to minimize the objective functions, which will be discussed in Section III-C. To guarantee a unique solution of the object's surface, integrability is enforced as in [10], where the gradients of surface heights are expressed as

$$
\left\{\begin{array}{l}
z_{x}=-\tan \theta_{n} \\
z_{y}=-\frac{\tan \phi_{n}}{\cos \theta_{n}}
\end{array} .\right.
$$

The shape of the human face is then modelled and represented as the height function $z=z(x, y)$.

\section{Implementation Details}

To minimize the objective function (13) or (14), a genetic algorithm (GA) is utilized similar to [29]. GA is a stochastic optimization method, which guides randomly selected solutions to evolve through a number of generations towards the global optimum and avoids differentiations of cost functions. Generally, it suffers less from being trapped into local minima than greedy optimization methods in the cost of efficiency. In experiments, it is found that GA operated in an acceptable efficiency on the HSD approach. Other optimization methods which are capable of overcoming local minima may also be used with further investigations on efficiency and precision levels. Because the focus of this paper is to propose an approach of modelling human faces with spatially varying diffuse and specular reflections, the comparison between optimization methods is out of its scope.

Details of GA in this proposed approach are as follows. All six parameters are represented in binary format and encoded as chromosomes for genetic algorithm with the following boundary settings. The normal directions $\theta_{n}$ and $\phi_{n}$ ranges are both $(-\pi / 2, \pi / 2)$, while in practice we eliminate the boundary effects by slightly reducing the range to $[-1.5,1.5]$. The reflectance coefficients $k_{a}, k_{d}$, and $k_{s}$ are all in the range of $[0,1]$ where zero indicates the surface is nonreflective and one indicates it is a perfect reflector. The specular exponent $n_{s}$ or $m_{s}$ is set within $[0.2,10]$ to allow a considerable range to vary. The parameters for a genetic algorithm are as follows. The population size is denoted as $\kappa_{p}$, the crossover rate is denoted as $\kappa_{c}$, the mutation rate is denoted as $\kappa_{m}$, the number of trials is denoted as $\kappa_{t}$, and the threshold of error is denoted as $\kappa_{e}$.

If the lighting conditions are not known a priori, the proposed HSD approach can still operate using light estimation as a preprocessing step. Although light estimation has been extensively studied, such as in [16], [21], [27], [33], and [34] which could be applied to assist our approach, we also propose a light estimation based on the specific problem of modelling faces as heterogeneous surfaces beyond Lambert. Since the lighting conditions are constant to all pixels in an image, light estimation is a global process over the whole face area. To reduce the dimensionality, a small number ( $p$, around 100) of sparse surface points $\left(\Omega=\left\{\left(x_{l}, y_{l}\right) \mid l=1,2, \ldots, p\right\}\right)$ are randomly selected for light estimation. The probability distributions of normal azimuth angle $\theta_{n}$ and zenith angle $\phi_{n}$ are assumed to be uniform with mean values $\theta_{n 0}$ and $\phi_{n 0}$. These two average angles are calculated from a generic 3-D face shape as

$$
\left\{\begin{array}{l}
\theta_{n 0}=\arctan \left(-\frac{\partial z_{0}}{\partial x}\right) \\
\phi_{n 0}=\arctan \left(-\frac{\partial z_{0}}{\partial y} \cos \theta_{n 0}\right)
\end{array} .\right.
$$

The ranges of $\theta_{n}$ and $\phi_{n}$ are $\left[\theta_{n 0}-\Delta_{\theta}, \theta_{n 0}+\Delta_{\theta}\right]$ and $\left[\phi_{n 0}-\right.$ $\left.\Delta_{\phi}, \phi_{n 0}+\Delta_{\phi}\right]$, respectively. The normal vector is then expressed as

$$
\boldsymbol{n}=\left(\sin \theta_{n} \cos \theta_{n}, \sin \phi_{n}, \cos \theta_{n} \cos \phi_{n}\right)^{T}
$$


TABLE I

(A) Estimated Directions AND (B) Light InTENSITIES USING Proposed Light Estimation Method. (A) Estimation ACCURACies on Lighting DiRECTIONS. (B) ESTIMATION RESUlTS ON LIGHT INTENSITIES (Avg. = Average value, S.D. = Standard deviation)

\begin{tabular}{|c|c|c|}
\hline Angle & Average error & $\begin{array}{c}\text { Standard } \\
\text { deviation }\end{array}$ \\
\hline$\theta_{i}$ & $3.55^{\circ}$ & $3.54^{\circ}$ \\
\hline$\phi_{i}$ & $9.60^{\circ}$ & $6.41^{\circ}$ \\
\hline
\end{tabular}

(a)

\begin{tabular}{|c||c|c|c|c|c|c|c|}
\hline \multirow{2}{*}{$I_{a}$} & \multicolumn{8}{|c|}{ Light number } \\
\cline { 2 - 8 } & 02 & 03 & 04 & 05 & 06 & 07 & 08 \\
\hline \hline Avg. & 167 & 156 & 107 & 121 & 160 & 158 & 189 \\
S.D. & 45.6 & 42.6 & 6.9 & 9.3 & 19.1 & 20.5 & 24.3 \\
\hline & \multicolumn{8}{|c|}{ Light number } \\
\cline { 2 - 8 }$I_{a}$ & 09 & 10 & 11 & 12 & 13 & 14 & 15 \\
\hline \hline Avg. & 181 & 133 & 184 & 160 & 132 & 129 & 107 \\
S.D. & 41.1 & 8.9 & 23.4 & 10.1 & 4.9 & 1.8 & 10.5 \\
\hline & \multicolumn{8}{|c|}{ Light number } \\
\cline { 2 - 8 }$I_{a}$ & 16 & 17 & 18 & 19 & 20 & 21 & 22 \\
\hline \hline Avg. & 134 & 125 & 108 & 153 & 183 & 161 & 129 \\
S.D. & 11.6 & 3.0 & 14.8 & 15.2 & 25.4 & 12.6 & 2.1 \\
\hline
\end{tabular}

(b)

Lighting conditions represented by $\theta, \phi, I_{i}$ and $I_{a}$ are then estimated by minimizing the following objective function with two other variable parameters $\theta_{n}$ and $\phi_{n}$ as

$$
E_{l}=\sum_{j \in(1, t)} \sum_{(x, y) \in \Omega}\left\{\begin{array}{c}
I_{j}-I_{a} k_{a} \\
-I_{i j}\left[k_{d}\left(\boldsymbol{n} \cdot \boldsymbol{l}_{j}\right)+k_{s}\left(\boldsymbol{r}_{j} \cdot \boldsymbol{v}\right)^{n_{s}}\right]
\end{array}\right\}^{2}
$$

for the Phong model.

We used the CMU-PIE database to test our light estimation method. In the experiment, we set $p=100$, and $\Delta_{\theta}=\Delta_{\phi}=$ $10^{\circ}$. The parameters for genetic algorithms are $\kappa_{p}=100, \kappa_{c}=$ 0.5 , and $\kappa_{n}=0.0002$. We found it was helpful to enlarge the population size and reduce the mutation rate as the chromosomes are generally longer than those in the face modelling process. The lighting directions given in the CMU-PIE database were used as ground truths and the lighting estimation results are listed in Table I. The estimated directions and intensities can be used in the subsequent face modelling process, if not known.

In practice, the set of gallery images inevitably contains spatial perturbations, which causes pixel misalignment in 3-D modelling. This effect usually results in noisy estimated reflectance coefficients and consequently the virtual views synthesized from these coefficients for face recognition are affected. To alleviate the effect of image perturbations in face recognition stage, a postprocessing for perturbation alleviation (PA) mechanism is proposed. Without loss of generality, the Phong model is incorporated into the perturbation alleviation processing. The reflectance coefficients are constrained locally by adding a smoothness function as

$$
E_{p a}^{j}(x, y)=\sum_{\Delta x=-1}^{1} \sum_{\Delta y=-1}^{1}\left\|\begin{array}{c}
k_{j}(x, y) \\
-k_{j-1}(x+\Delta x, y+\Delta y)
\end{array}\right\|^{2}
$$
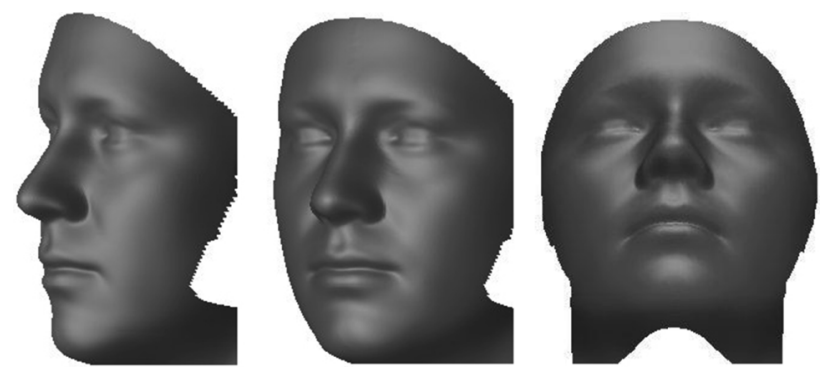

Fig. 2. Ground-truth of 3-D face shape used in the experiment.

where $j$ denotes the iteration number and $\boldsymbol{k}=\left\{k_{a}, k_{d}, k_{s}, n_{s}\right\}^{T}$ are the vectors formed by the reflectance coefficients of the Phong model. This penalty constrains the optimization iteration of (13) to alleviate reflectance perturbation caused by misalignment. Combining (22) and (13), the objective function is then expressed as

$$
E_{r c}(x, y)=E_{p}(x, y)+w E_{p a}^{j}(x, y)
$$

where $w$ is the weight balancing the modelling sensitivity and the perturbation alleviation. The estimated reflectance coefficients from the shape modelling are used as the initial parameters of $\boldsymbol{k}_{j}$. In practice, we choose $w=20$ and the iteration generally converges after $j>5$. This processing is not necessary to synthetic gallery data which are free of image perturbations. Thus, only the third experiment on real images employed this perturbation alleviation processing.

\section{EXPERIMENTS}

We evaluated the proposed HSD 3-D surface approximation approach on synthetic and real face images in three experiments. The first experiment is to compare the face modelling results under the Lambertian assumption and beyond the Lambertian assumption. In the second experiment, we investigate the effectiveness of extending the surface reflectance assumption from homogeneity to heterogeneity in the context of face modelling. The proposed HSD approach was compared to the typical photometric stereo which is based on a homogeneous assumption on specular reflectivity. Both of the two comparisons are made by measuring modelling accuracies between ground truth models and estimated models. Finally, face recognition across pose on real face images from the publicly available database is performed, in the third experiment, to evaluate how effective the HSD approach works in face recognition across pose, compared to two current face recognition systems dealing with pose variations [14], [15].

\section{A. Modelling Under and Beyond Lambertian Assumption}

The average face shape from USF human ID 3-D database [5] was used to render synthetic gallery images with the same lighting directions as specified in CMU-PIE database [36]. For fair comparison, we intentionally used different reflectance models to render the gallery images and to estimate $3-\mathrm{D}$ face 


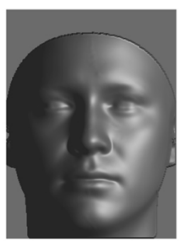

(a)

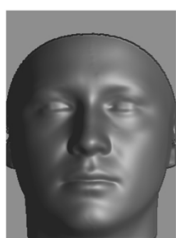

(b)

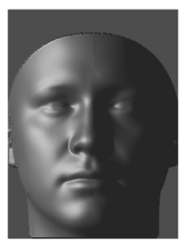

(c)

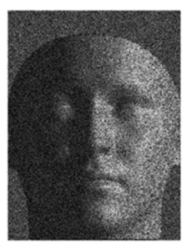

(d)

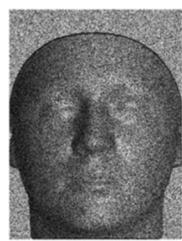

(e)

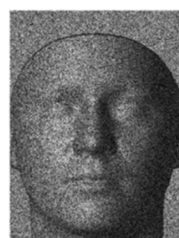

(f)

Fig. 3. Examples of the gallery images synthesized using Torrance-Sparrow model and the average 3-D face shape [5] shown in Fig. 2. (a)-(c) Homogeneous gallery images whose ambient, diffuse, and specular reflectivities are all uniform. (d)-(f) Heterogeneous gallery images whose ambient, diffuse, and specular reflectivities are all spatially varying.

models, respectively. The rendering model is a simplified Torrance-Sparrow model without Fresnel equation and the models used in estimations are Lambertian model and Phong model, respectively. The homogeneous gallery images were synthesized with the ambient, diffuse, and specular reflectance coefficients $k_{a}=k_{d}=k_{s}=0.4$, surface roughness $m_{s}=0.5$, ambient light intensity $I_{a}=100$, and directional light intensity $I_{i}=150$. For the heterogenous gallery images, the ambient, diffuse, and specular reflectance coefficients and the surface roughness were all randomly allocated obeying the uniform probability distributions within the ranges $k_{a}, k_{d}, k_{s} \in[0,1]$ and $m_{s} \in[0.2,10]$. Other parameters were the same as generating the first set of gallery images with homogeneous surface restrictions. The resolutions of these two sets of gallery images are both $250 \times 340$ pixels. Fig. 2 shows the average face shape used as the ground truth for generating the synthetic gallery images and the modelling experiments and Fig. 3 shows three example gallery images from each set of the synthetic images.

Within the proposed image-based 3-D modelling framework, the Lambertian model and Phong model were incorporated in the modelling processes, which were applied on the two sets of gallery images, respectively. The optimization parameters are $\kappa_{p}=50, \kappa_{m}=0.02, \kappa_{c}=0.6, \kappa_{t}=10^{4}$, and $\kappa_{e}=1.5$. The estimated 3-D face shapes from the homogeneous and heterogeneous gallery images are shown in Figs. 4 and 5, respectively. Different from [6], [20], these face shapes are modelled purely from 2-D images without using prior 3-D knowledge of human faces. The average cosine value between the ground truth normal directions (denoted as "gnd") and the estimated normal directions (denoted as "est") is used to measure the shape modelling accuracy as

$$
\cos \left(\boldsymbol{n}_{\text {gnd }}, \boldsymbol{n}_{\text {est }}\right)=\frac{1}{N} \sum_{(x, y)} \cos \left(\boldsymbol{n}_{\text {gnd }}(x, y), \boldsymbol{n}_{\text {est }}(x, y)\right)
$$

where $N$ is the number of image pixels, $\boldsymbol{n}_{\text {gnd }}(x, y)$ is the ground-truth normal direction at point $(x, y)$, and $\boldsymbol{n}_{\text {est }}(x, y)$ is its estimate [55]. The average angle value of the errors is then calculated from the cosine value. The shape modelling accuracies are summarized in Table II in both average cosine values and angular values as the measures of the modelling errors.

On both homogeneous and heterogeneous gallery faces, the 3-D modelling using the Phong model considering both diffuse and specular reflections is significantly superior to those using Lambertian model considering only diffuse reflection.
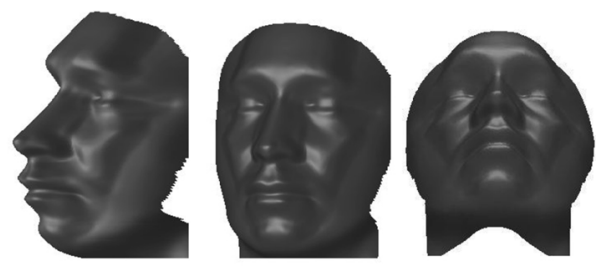

(a)
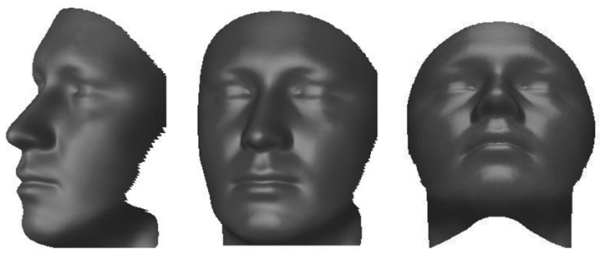

(b)

Fig. 4. Estimated results using the proposed approach based on (a) Lambertian model and (b) Phong model from the homogeneous gallery images.
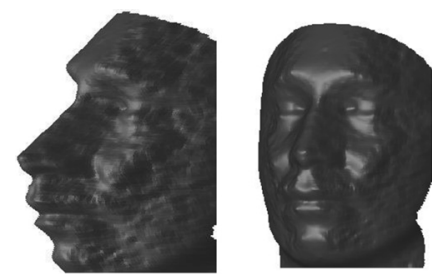

(a)
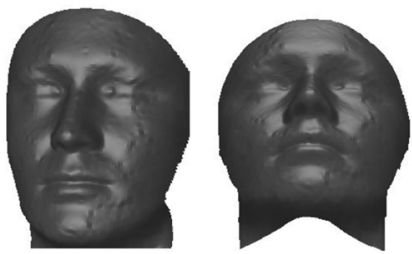

(b)
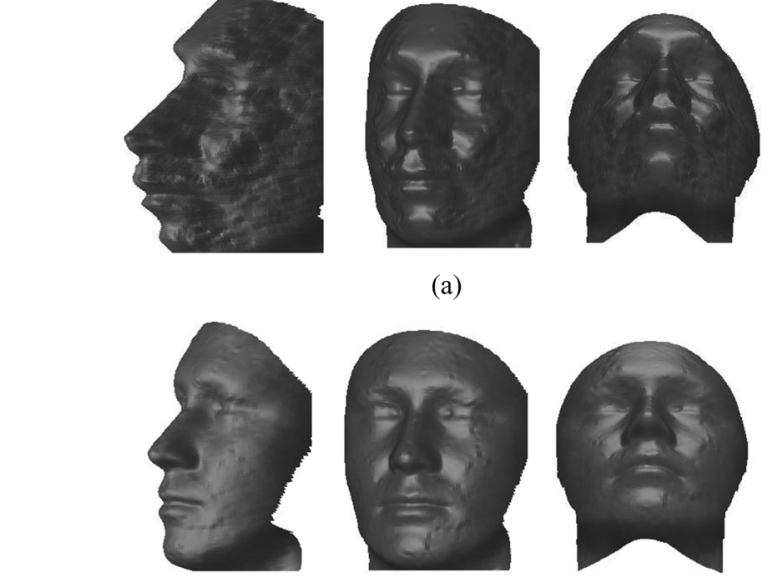

Fig. 5. Modelling results using the proposed HSD approach based on (a) Lambertian model and (b) Phong model from the heterogeneous gallery images [Fig. 3(d)-(f)].

Compared to 3-D modelling using the Lambertian model, the modelling using the Phong model reduced modelling error by $54.0 \%$ on the homogeneous gallery faces and by $60.0 \%$ on the heterogeneous gallery faces. Because the gallery images were generated using the Torrance-Sparrow model, the comparison is not biased to either of the parties, which demonstrates the effectiveness and necessity of 3-D face modelling beyond the Lambertian assumption. The Lambertian-based method generated suboptimal results because it inappropriately fitted the 
TABLE II

Modelling Accuracies of Proposed Framework Using Different Reflectance Models in Forms of cos $\left(\boldsymbol{n}_{\text {gnd }}, \boldsymbol{n}_{\text {est }}\right)$ and Average Angle

\begin{tabular}{|c||c|c|}
\hline Modelling approaches & $\begin{array}{c}\text { Modelling under the } \\
\text { Lambertian assumption }\end{array}$ & HSD \\
\hline \hline $\begin{array}{c}\text { Homogeneous gallery faces with uniform specular, diffuse, } \\
\text { and ambient reflectivities }\end{array}$ & $0.9521\left(17.80^{\circ}\right)$ & $\begin{array}{c}0.9898 \\
\left(8.18^{\circ}\right)\end{array}$ \\
\hline $\begin{array}{c}\text { Heterogeneous gallery faces with spatially varying specular, } \\
\text { diffuse, and ambient reflectivities }\end{array}$ & $0.9286\left(21.78^{\circ}\right)$ & $\begin{array}{c}0.9884 \\
\left(8.72^{\circ}\right)\end{array}$ \\
\hline
\end{tabular}

diffuse-only reflectance model to a face surface which exhibits significant specularity. Without the inclusion of a specular component, the Lambertian-based method forces incorrect shape changes to approximate intensity changes caused by specular reflection. Consequently, the estimated 3-D face shape is deviated from the ground truth shape. It can be concluded that the 3-D modelling is sensitive to the inclusion of specular reflection modelling, but not sensitive to the specific expression of specular reflection modelling. Though Phong model and Torrance-Sparrow model are different in the specular reflection expressions, the Phong specular component is still a good approximation of the Torrance-Sparrow specular component. In the real photographs, both Phong and Torrance-Sparrow models are approximations to the real-world reflections which could be more complicated. The capability of using one reflection model to approximate scenes rendered by different unknown reflection models is helpful to simplify the problem and computation within the proposed 3-D modelling framework.

The modelling results on heterogeneous data are less accurate than the results on homogeneous data. One possible reason is that when creating the heterogeneous gallery images by randomly allocating reflectance coefficients, some points may be singular for inverse estimation. Singularity occurs when a surface point has small values on both diffuse and specular reflectance coefficients (i.e., $k_{d} \sim 0$ and $k_{s} \sim 0$ ). In such a case, the pixel intensity is merely ambient reflection and no shape information can be reversely estimated. Because the same gallery faces were used for both modelling processes, the influences of singularity should be similar. However, the experimental results reveal that the modelling error increases from homogeneous data to heterogeneous data are $6.6 \%$ (from $8.18^{\circ}$ to $8.72^{\circ}$ ) when using Phong model, and $22.4 \%$ (from $17.80^{\circ}$ to $21.78^{\circ}$ ) when using the Lambertian model, respectively. This finding indicates that neglecting specular reflection has more severe effects on heterogeneous surfaces than on homogeneous surfaces and it is more preferable to incorporate reflectance models beyond the Lambertian assumption when the object surface reflectivity is spatially varying.

\section{B. Three-Dimensional Modelling Under and Beyond Homogeneous Assumption}

This section investigates the effect of modelling the specular reflectance coefficient as heterogeneous compared to modelling it as homogeneous under standard photometric stereo settings. The proposed HSD approach was compared to typical homogeneous specular photometric stereo (HSPS) approach. Because we intended to compare the methods assuming homogeneous specular reflectivity and assuming heterogeneous specular reflectivity, the two gallery faces were synthesized using uniform
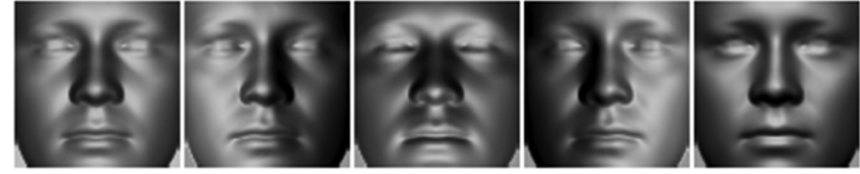

(a)
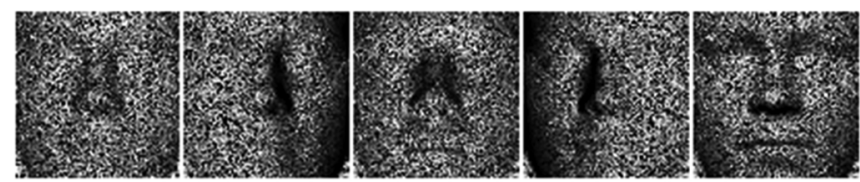

(b)

Fig. 6. Example gallery images synthesized using the Torrance-Sparrow model and the average 3-D face shape [5] for 3-D face modelling experiments. (a) Homogeneous face whose reflectance coefficients are uniform and (b) heterogeneous gallery face whose reflectance coefficients are all spatially varying.

TABLE III

LIGHTING DIRECTIONS (IN DEGREES) USED FOR RENDERING GALLERY IMAGES

\begin{tabular}{|c||ccccccc|}
\hline Light & 0 & 1 & 2 & 3 & 4 & 5 & 6 \\
\hline Yaw & 0 & -15 & -15 & -15 & 0 & 0 & 15 \\
Tile & 0 & -15 & 0 & 15 & -15 & 15 & -15 \\
\hline Light & 7 & 8 & 9 & 10 & 11 & 12 & 13 \\
\hline Yaw & 15 & 15 & -30 & -30 & -30 & -15 & -15 \\
Tilt & 0 & 15 & -15 & 0 & 15 & -30 & 30 \\
\hline Light & 14 & 15 & 16 & 17 & 18 & 19 & 20 \\
\hline Yaw & 0 & 0 & 15 & 15 & 30 & 30 & 30 \\
\hline Tilt & -30 & 30 & -30 & 30 & -15 & 0 & 15 \\
\hline
\end{tabular}

reflectance coefficients and spatially varying reflectance coefficients, respectively. These two gallery sets were generated using the simplified Torrance-Sparrow model where Fresnel equation and geometric attenuation factor were neglected. The HSD approach differs from the HSPS approach in two distinct aspects: 1) the HSD approach allows both diffuse and specular reflectance coefficients to vary spatially along the surface while the HSPS approach only allows the diffuse reflectance coefficient to vary spatially and keeps the specular reflectance coefficient and surface roughness homogeneous; and 2) the HSD method locally optimizes both shape and reflectance coefficients while the HSPS approach iteratively and sequentially optimizes five sets of the parameters for the entire image, i.e., lights, shape, heterogeneous diffuse reflectance coefficients, homogeneous specular reflectance coefficient, and homogeneous surface roughness. The image size was $80 \times 80$ for both methods in consideration of the slow speed of the HSPS. The rendering parameters were $I_{i}=200, k_{d}=k_{s}=m_{s}=0.5$ for homogeneous gallery faces and $I_{i}=200, k_{d} \in[0,1]$, $k_{s} \in[0,1]$, and $m_{s} \in[0.2,10]$ for heterogeneous gallery faces. The lighting directions are listed in Table III and the examples of the gallery face images are shown in Fig. 6. For HSPS, the 
TABLE IV

Modelling Accuracies of HSPS Approach AND Proposed HSD Approach on Homogeneous and Heterogeneous Gallery FaCes, In Forms of $\cos \left(\boldsymbol{n}_{\text {gnd }}, \boldsymbol{n}_{\text {est }}\right)$ AND AVERAGE ANGLE

\begin{tabular}{|c|c|c|}
\hline Modelling approaches & $\begin{array}{c}\text { The HSPS } \\
\text { approach }\end{array}$ & $\begin{array}{c}\text { The proposed } \\
\text { HSD approach }\end{array}$ \\
\hline \hline Homogeneous gallery face with & 0.9872 & 0.9932 \\
$\left(9.15^{\circ}\right)$ & $\left(6.65^{\circ}\right)$ \\
\hline uniform specular and diffuse reflectivities & 0.5991 & 0.9928 \\
Heterogeneous gallery face with & $\left(53.19^{\circ}\right)$ & $\left(6.88^{\circ}\right)$ \\
\hline
\end{tabular}

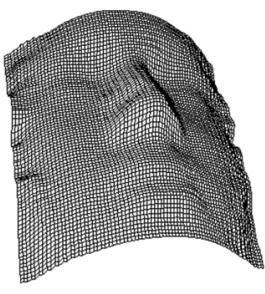

(a)

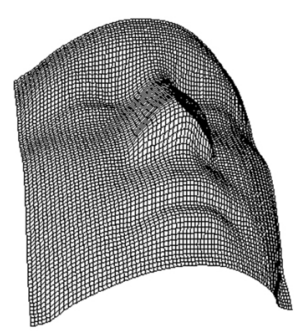

(c)

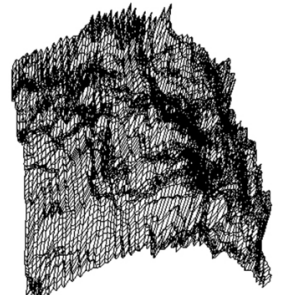

(b)

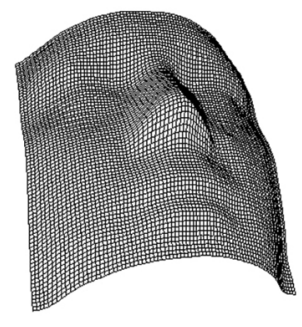

(d)
Fig. 7. Modelling results of the traditional HSPS approach and proposed HSD approach. (a) Estimated shape using the HSPS approach on homogeneous data. (b) Estimated shape using the HSPS approach on heterogeneous data. (c) Estimated shape using the HSD approach on homogeneous data. (d) Estimated shape using the HSD approach on heterogeneous data.

parameters were set the same as in [12]. For HSD, the Phong model is used and the optimization parameters were set the same as in Experiment 1. Examples of the estimated faces are displayed in Fig. 7 and the model accuracies are summarized in Table IV.

From Table IV, the modelling results of the HSPS approach and the HSD approach on homogeneous gallery faces are comparable with the HSD approach achieving slightly higher accuracy than the HSPS approach. For heterogeneous gallery faces, however, the HSPS approach assuming homogeneous specular reflectivity could not converge to a sound modelling result while the HSD approach performed nearly as well as on the homogeneous gallery faces. The reason is believed to be the homogeneity assumption of the HSPS approach on specular reflectance coefficients, which resulted in the HSPS approach incapable of handling the heterogeneous surface reliably. The HSD approach, on the other hand, performed consistently well on both homogeneous and heterogeneous surfaces. These experimental results have shown the necessity of the 3-D human face modelling to approximate face surfaces with spatially varying specular reflectivity as well as diffuse reflectivity. With these more accurate assumptions, 3-D face modelling approaches can perform reliably and achieve higher accuracy on estimated shapes. Specifically for image-based 3-D modelling methods, the assumptions and modelling on surface reflectivity

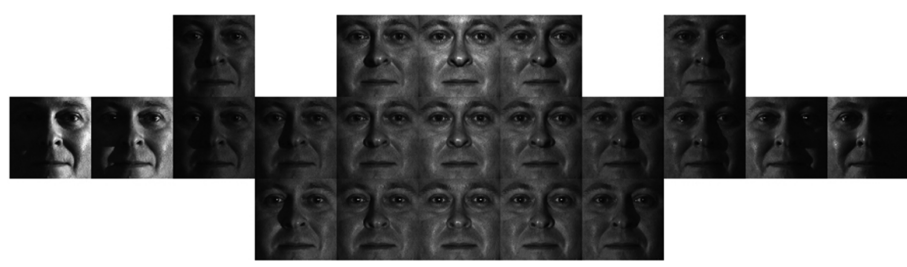

Fig. 8. Examples of the real gallery images from PIE database used for 3-D modelling.

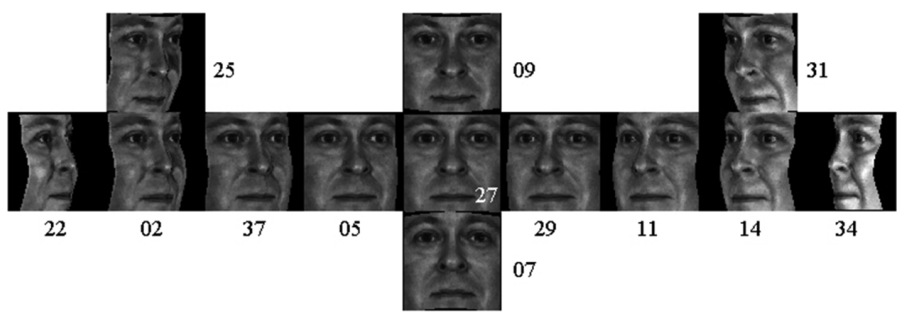

Fig. 9. Virtual views in 13 different poses synthesized from the estimated personalized 3-D face model of the same person as shown in Fig. 8 using the proposed HSD modelling approach.

affect not only reflectivity estimation, but also shape estimation as shown in this experiment. Therefore, accurate approximation of the image formation process is highly recommended to shape estimation techniques from image intensities.

\section{Face Recognition Experiments Across Pose}

In the third experiment, the HSD approach was tested on real face images from CMU-PIE database [36]. This publicly available database contains more than 40000 face pictures of 68 people taken in different poses $(\mathrm{P})$, under various illuminations (I) and with different expressions (E). A subset containing 13 different poses and 21 different lighting conditions was used in the experiment, to test the HSD approach in a face-recognition-across-pose setting. The gallery images were faces under different illuminations (Fig. 8) while probe images to be recognized were rotated faces in poses ranging from $-62^{\circ}$ to $66^{\circ}$ in yaw and $-13^{\circ}$ to $13^{\circ}$ in tilt. The lighting estimation described in Section III-C was used to provide the illumination conditions of the gallery images for the HSD modelling approach. The optimization parameters were $\kappa_{p}=50, \kappa_{m}=0.02, \kappa_{c}=0.6$, $\kappa_{t}=10^{4}$, and $\kappa_{e}=1.5$, and the Phong model was selected as the reflectance model. The estimated 3-D face models were then rotated and projected to synthesize virtual face views for recognition as shown in Fig. 9.

Using local binary patterns (LBP) [1] as the classifier, face recognition experiments were conducted on the real views and the corresponding virtual views synthesized using the proposed 
TABLE V

Average Recognition Rates (\%) of Proposed HSD Approach Compared With Other Approaches

\begin{tabular}{|c||c|c|c|c|c|}
\hline & Baseline & ELF & PDM & ICM & HSD \\
\hline \hline $\begin{array}{c}\text { Average recognition rate } \\
\text { over 8 poses }\end{array}$ & - & - & 69.38 & - & $\mathbf{8 4 . 2 2}$ \\
\hline $\begin{array}{c}\text { Average recognition rate } \\
\text { over 12 poses }\end{array}$ & 59.20 & 63.42 & - & 72.72 & $\mathbf{8 7 . 3 3}$ \\
\hline
\end{tabular}

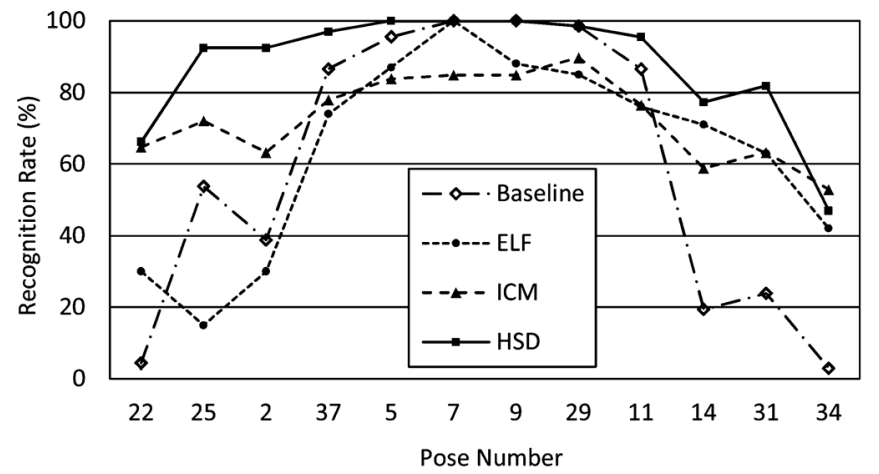

(a)

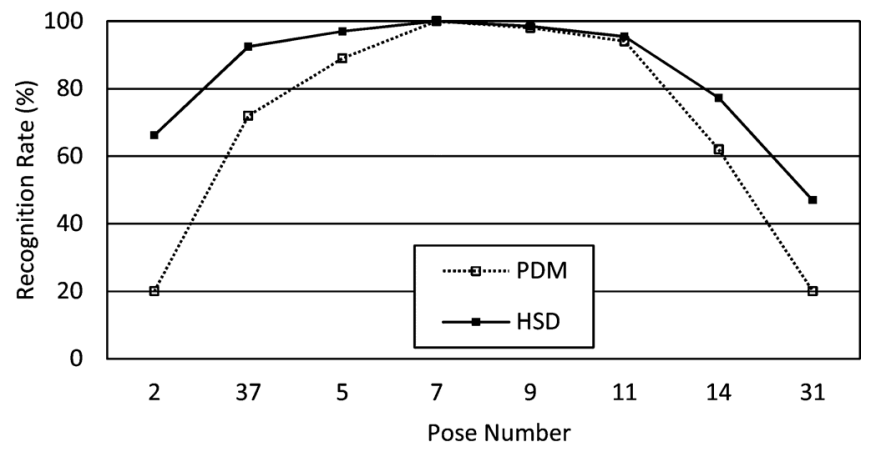

(b)

Fig. 10. Face recognition performances of the proposed HSD approach compared with (a) baseline approach (Base), Eigen-light field (ELF), illumination cone models (ICM), and (b) point distribution models (PDM). Pose numbers in the charts are from the CMU-PIE database as illustrated in Fig. 9.

HSD approach. The recognition directly using frontal images as gallery views without synthesizing virtual views (denoted as "Baseline") was chosen as the baseline. The illumination cone model (ICM) [11] was implemented and the synthesized virtual views were used in the same face recognition framework for comparison. The reported recognition rates of Eigen light-fields (ELF) [15] and point distribution model (PDM) [14], two of the recent pose-invariant face recognition methods with thorough experimental results on the CMU-PIE database, were also included for comparison. For HSD and ICM methods, the gallery has 21 frontal images per subject as shown in Fig. 8. For each face, a 3-D face model was recovered and virtual views in different poses were synthesized (as shown in Fig. 9 for HSD approach). These virtual views were then used as a virtual gallery for recognizing real face images in different poses. The ELF and PDM methods required multiple training subjects with a single gallery image per pose per subject. Thirty-four subjects were used for training and the images of the remaining 34 subjects were used as a gallery for recognition. The recognition rates of the proposed HSD approach and other approaches were presented in Table V and Fig. 10 for comparison.
The experimental results show that the proposed HSD approach significantly improved the face recognition performance against the direct matching baseline (Baseline) by $28.13 \%$. Note that HSD greatly improved the recognition accuracy over Baseline in every pose. Compared to the reported recognition rates of ELF and PDM, the HSD approach achieved higher recognition rates as well. For all 12 poses, HSD outperformed ELF by 23.91\%. Because PDM only experimented on eight poses (i.e., pose 2, 37, 5, 7, 9, 11, 14, 31 as listed in Fig. 10), the comparison of average recognition rates is conducted on these eight poses where HSD achieved 84.22\% accuracy over PDM's $69.38 \%$. The improvements were made by including more frontal images when estimating personalized 3-D face models but without training steps as in ELF and PDM. Comparing to recognition results of ICM which is a similar approach to HSD except considering diffuse reflection only, the proposed HSD approach also performed significantly better with an improvement of $14.61 \%$ in average recognition rate.

\section{Modelling Speed}

An experiment was conducted to compare the modelling speeds of the proposed HSD approach and the benchmark approaches of ICM and HSPS. All approaches were implemented using Microsoft Visual $\mathrm{C}++2010$ without parallel computing programming and tested on a Dell Latitute E6500 laptop computer with Intel Core 2Duo CPU at 2.66 GHz and 4 GB DDR2 RAM. For the HSD approach, the average computational time of modelling one face as in Figs. 7 and 9 for Experiments 2 and 3 (Sections IV-B and IV-C) is $15 \mathrm{~min} 47 \mathrm{~s}$ (947 s). For the ICM approach, the average runtime of modelling one face for Experiment 3 (Section IV-C) is $2 \min 35 \mathrm{~s}(155 \mathrm{~s})$, and it takes the HSPS approach 2 hours $12 \min (7941 \mathrm{~s})$ to obtain the results as in Fig. 7 for Experiment 2 (Section IV-B). The proposed HSD approach is slower than the Lambertian-only ICM approach due to its more complex heterogeneous specular reflection considerations but both faster and more accurate than the non-Lambertian HSPS approach. As the modelling process is generally performed offline, modelling speed is not as an important issue as accuracy in many face recognition applications. Due to the independent processing of different local pixels in the HSD approach, the algorithm can be implemented in a distributed manner to increase its speed for real applications.

\section{CONCLUSIONS AND Future WORK}

Human faces are complex surfaces which exhibit both heterogeneous specular and diffuse reflectivities. Pose variations are a prominent challenge in face recognition. Existing 3-D modelling techniques for face recognition across poses are limited to and built on either the Lambertian surface assumption or 
the homogeneous reflectance assumption, which result in suboptimal 3-D shape and surface models. In this paper, a novel HSD 3-D surface approximation approach has been proposed to model 3-D human faces for face recognition across poses. From a small number of face images under different lighting conditions for each face, a personalized 3-D face model can be estimated from 2-D images without sacrificing the variability of both diffuse and specular reflectance coefficients. Instead of iteratively optimizing shape and reflectance parameters in traditional 3-D modelling techniques, we proposed a new optimization mechanism which locally searches optimal surface orientations and reflectance coefficients to achieve plausible modelling results. It overcomes the shape distortions caused by neglecting specular reflection component and computational instability due to homogeneity constraints on heterogeneous face surfaces. The estimated shape and surface reflectivity properties are then used in rendering face views under different viewing directions for recognizing faces in varying poses.

The proposed HSD approach was evaluated at three different experimental levels of: 1) comparing face modellings under and beyond the Lambertian assumption; 2) comparing face modellings under and beyond the homogeneous assumption; and 3) performing face modelling and recognition using real face images from the CMU-PIE database. The HSD approach achieved a higher level of performances in 3-D modelling accuracy, algorithm reliability, and face recognition accuracy. These results show that face modelling and recognition beyond Lambert and homogeneity is a feasible and better solution to pose-invariant face recognition.

This paper demonstrated the effectiveness and superiority of heterogeneous specular and diffuse surface approximation for face modelling and recognition. A number of future works could be important along this research direction to apply the proposed method in real applications. For example, lighting configurations can be further reduced and minimized to make the data acquisition easier and more practical. The proposed surface approximation can be expanded to other experiment setups such as shape-from-shading, stereo vision, etc., which cover broader application scenarios. Prior knowledge of human face structures can be incorporated into the proposed modelling framework to regulate the variations of face shape and surface reflective coefficients. Hence, the number of input face images can be reduced and existing face image databases could be made suitable for face recognition applications. Other interesting research domains include general object surface approximation, nonrigid 3-D face modelling, and face recognition in 3-D domain considering surface reflective property.

\section{ACKNOWLEDGMENT}

The authors would like to thank T.-J. Cham for help with obtaining the PIE database, B. Zhang for providing the algorithm of local binary patterns for recognition tests, and E. Hancock for providing constructive suggestions on the manuscript.

\section{REFERENCES}

[1] T. Ahonen, A. Hadid, and M. Pietikäinen, "Face description with local binary patterns: Application to face recognition," IEEE Trans. Pattern Anal. Mach. Intell., vol. 28, no. 12, pp. 2037-2041, Dec. 2006.
[2] S. Barsky and M. Petrou, "The 4-source photometric stereo technique for three-dimensional surfaces in the presence of highlights and shadows," IEEE Trans. Pattern Anal. Mach. Intell., vol. 25, no. 10, pp. 1239-1252, Oct. 2003.

[3] R. Basri and D. W. Jacobs, "Lambertian reflectance and linear subspaces," IEEE Trans. Pattern Anal. Mach. Intell., vol. 25, no. 2, pp. 218-233, Feb. 2003.

[4] R. Basri, D. Jacobs, and I. Kemelmacher, "Photometric stereo with general, unknown lighting," Int. J. Comput. Vis., vol. 72, no. 3, pp. 239-257, 2007.

[5] V. Blanz and T. Vetter, "A morphable model for the synthesis of 3D faces," in Proc. SIGGRAPH, 1999, pp. 187-194.

[6] V. Blanz and T. Vetter, "Face recognition based on fitting a 3D morphable model," IEEE Trans. Pattern Anal. Mach. Intell., vol. 25, no. 9, pp. 1063-1074, Sep. 2003.

[7] J. F. Blinn, "Models of light reflection for computer synthesized pictures," in Proc. SIGGRAPH, 1977, pp. 192-198.

[8] P. Debevec, T. Hawkins, C. Tchou, H. P. Duiker, W. Sarokin, and M. Sagar, "Acquiring the reflectance field of a human face," in Proc. SIGGRAPH, 2000, pp. 145-156.

[9] J. D. Foley, A. van Dam, S. K. Feiner, and J. F. Hughes, Computer Graphics: Principles and Practice, 2nd ed. Boston, MA: AddisonWesley, 1995.

[10] R. T. Frankot and R. Chellappa, "A method for enforcing integrability in shape from shading algorithms," IEEE Trans. Pattern Anal. Mach. Intell., vol. 10, no. 4, pp. 439-451, Apr. 1988.

[11] A. S. Georghiades, P. N. Belhumeur, and D. J. Kriegman, "From few to many: Illumination cone models for face recognition under variable lighting and pose," IEEE Trans. Pattern Anal. Mach. Intell., vol. 23, no. 6, pp. 643-660, Jun. 2001.

[12] A. S. Georghiades, "Recovering 3-D shape and reflectance from a small number of photographs," in Proc. EUROGRAPH, 2003.

[13] A. S. Georghiades, "Incorporating the Torrance and Sparrow model of reflectance in uncalibrated photometric stereo," in Proc. ICCV, 2003, vol. 2, pp. 816-823.

[14] D. González-Jiménez and J. L. Alba-Castro, "Toward pose-invariant 2-D face recognition through point distribution models and facial symmetry," IEEE Trans. Info. Foren. Sec., vol. 2, no. 3, pp. 413-429, Jun. 2007.

[15] R. Gross, I. Matthews, and S. Baker, “Appearance-based face recognition and light-fields," IEEE Trans. Pattern Anal. Mach. Intell., vol. 26, no. 4, pp. 449-465, Apr. 2004.

[16] K. Hara, K. Nishino, and K. Ikeuchi, "Light source position and reflectance estimation from a single view without the distant illumination assumption," IEEE Trans. Pattern Anal. Mach. Intell., vol. 27, no. 4, pp. 493-505, Apr. 2005.

[17] H. Hayakawa, "Photometric stereo under a light source with arbitrary motion," JOSA-A, vol. 11, no. 11, pp. 3079-3089, 1994.

[18] X. D. He, K. E. Torrance, F. X. Sillion, and D. P. Greenberg, "A comprehensive physical model for light reflection," Proc. SIGGRAPH 91 (Computer Graphics), vol. 25, pp. 175-186, 1991.

[19] B. K. P. Horn and M. J. Brooks, Shape From Shading. Boston, MA MIT Press, 1989.

[20] D. Jiang, Y. Hu, S. Yan, L. Zhang, H. Zhang, and W. Gao, "Efficient 3D reconstruction for face recognition," Pattern Rec., vol. 38, no. 6 , pp. 787-798, 2005.

[21] M. Kanbara and N. Yokoya, "Real-time estimation of light source environment for photorealistic augmented reality," in Proc. 17th Int. Conf. Pattern Recognition, 2004, vol. 2, pp. 911-914.

[22] J. Lambert, Photometria Sive De Mensura Et Gradibus Luminus Colorum et Umbrae. : Eberhard Klett, 1760.

[23] K.-C. Lee, J. Ho, and D. J. Kriegman, "Acquiring linear subspaces for face recognition under variable lighting," IEEE Trans. Pattern Anal. Mach. Intell., vol. 27, no. 5, pp. 684-698, May 2005.

[24] H. Nakai, Y. Manabe, and S. Inokuchi, "Simulation and analysis of spectral distributions of human skin," in Proc. ICPR, 1998, vol. 2, pp. $1065-1067$.

[25] C. S.-L. Ng and L. Li, "A multi-layered reflection model of natural human skin," Computer Graphics Int., pp. 249-256, 2001.

[26] F. E. Nicodemus, J. C. Richmond, J. J. Hsia, I. W. Ginsberg, and T. Limperis, "Geometrical considerations and nomenclature for reflectance," NBS Monograph (U.S.), Oct. 1977.

[27] W. M. Pang, T. T. Wong, and P. A. Heng, "Estimating light vectors in real time," IEEE Computer Graphics Applicat., vol. 24, no. 3, pp. 36-43, Mar. 2004.

[28] B. T. Phong, "Illumination for computer generated pictures," Comms. $A C M$, vol. 18 , no. 6 , pp. $311-317,1975$. 
[29] S. J. Preece and E. Claridge, "Spectral filter optimization for the recovery of parameters which describe human skin," IEEE Trans. Pattern Anal. Mach. Intell., vol. 26, no. 7, pp. 913-922, Jul. 2004.

[30] R. Ramamoorthi, "Analytic PCA construction for theoretical analysis of lighting variability in images of a Lambertian object," IEEE Trans. Pattern Anal. Mach. Intell., vol. 24, no. 10, pp. 1322-1333, Oct. 2002.

[31] S. Romdhani and T. Vetter, "Estimating 3D shape and texture using pixel intensity, edges, specular highlights, texture constraints and a prior," in Proc. IEEE Conf. CVPR, 2005, vol. 2, pp. 986-993.

[32] S. Romdhani, J. Ho, T. Vetter, and D. J. Kriegman, "Face recognition using 3-D models: Pose and illumination," Proc IEEE, vol. 94, no. 11, pp. 1977-1999, 2006.

[33] D. Samaras and D. Metaxas, "Incorporating illumination constraints in deformable models for shape from shading and light direction estimation," IEEE Trans. Pattern Anal. Mach. Intell., vol. 25, no. 2, pp. 247-264, 2003.

[34] I. Sato, Y. Sato, and K. Ikeuchi, "Illumination from shadows," IEEE Trans. Pattern Anal. Mach. Intell., vol. 25, no. 3, pp. 290-300, Mar. 2003.

[35] S. A. Shafer, "Using color to separate reflection components," Color Res. Applicat., vol. 10, no. 4, pp. 210-218, 1985.

[36] T. Sim, S. Baker, and M. Bsat, "The CMU pose, illumination, and expression database," IEEE Trans. Pattern Anal. Mach. Intell., vol. 25, no. 12, pp. 1615-1618, Dec. 2003.

[37] F. Solomon and K. Ikeuchi, "Extracting the shape and roughness of specular lobe objects using four light photometric stereo," IEEE Trans. Pattern Anal. Mach. Intell., vol. 18, no. 4, pp. 449-454, Apr. 1996.

[38] H. D. Tagare and R. J. P. deFigueiredo, "A theory of photometric stereo for a class of diffuse non-Lambertian surfaces," IEEE Trans. Pattern Anal. Mach. Intell., vol. 13, no. 2, pp. 133-152, Feb. 1991.

[39] R. T. Tan and K. Ikeuchi, "Separating reflection components of textured surfaces using a single image," IEEE Trans. Pattern Anal. Mach. Intell., vol. 27, no. 2, pp. 178-193, Feb. 2005.

[40] K. Torrance and E. M. Sparrow, "Theory for off-specular reflection from roughened surfaces," J. Optical Soc. America, vol. 56, no. 7, pp. 916-925, 1967.

[41] M. J. C. Van Gemert, S. L. Jacques, H. J. C. M. Sterenborg, and W. M. Star, "Skin optics," IEEE Trans. Biomed. Eng., vol. 36, no. 12, pp. 1146-1154, Dec. 1989.

[42] T. Weyrich, W. Matusik, H. Pfister, B. Bickel, C. Donner, C. Tu, J. McAndless, J. Lee, A. Ngan, H. W. Jensen, and M. Gross, "Analysis of human faces using a measurement-based skin reflectance model," Proc. SIGGRAPH 2006 (Computer Graphics), pp. 1013-1024, 2006.

[43] R. J. Woodham, R. Nevatia, and R. Nevatia, "Photometric stereo: A reflectance map technique for determining surface orientation from image intensity," in Proc. SPIE, 1978, vol. 155, pp. 136-143.

[44] R. J. Woodham, "Photometric method for determining surface orientation from multiple images," Optical Eng., vol. 19, no. 1, pp. 139-144, 1980.

[45] T.-P. Wu, K.-L. Tang, C.-K. Tang, and T.-T. Wong, "Dense photometric stereo: A Markov random field approach," IEEE Trans. Pattern Anal. Mach. Intell., vol. 28, no. 11, pp. 1830-1846, Nov. 2006.

[46] A. L. Yuille, D. Snow, R. Epstein, and P. N. Belhumeur, "Determining generative models of objects under varying illumination: Shape and albedo from multiple images using SVD and integrability," Int. J. Comput. Vis., vol. 35, no. 3, pp. 203-222, 1999.

[47] L. Zhang and D. Samaras, "Face recognition from a single training image under arbitrary unknown lighting using spherical harmonics," IEEE Trans. Pattern Anal. Mach. Intell., vol. 28, no. 3, pp. 351-363, Mar. 2006.
[48] R. Zhang, P.-S. Tsai, J. E. Cryer, and M. Shah, "Shape-from-shading: A survey," IEEE Trans. Pattern Anal. Mach. Intell., vol. 21, no. 8, pp. 690-706, Aug. 1999.

[49] X. Zhang, Y. Gao, and M. K. H. Leung, "Multilevel quadratic variation minimization for 3D face modeling and virtual view synthesis," in Proc. Int. Multimedia Model. Conf., 2005, pp. 132-138.

[50] X. Zhang, Y. Gao, and B.-L. Zhang, "Recognising rotated faces from two orthogonal views in mugshot databases," in Proc. ICPR, 2006, vol. 1, pp. $195-198$.

[51] X. Zhang, Y. Gao, and M. K. H. Leung, "Recognizing rotated faces from frontal and side views: An approach towards effective use of mugshot databases," IEEE Trans. Info. Foren. Sec., vol. 3, no. 4, pp. 684-697, 2008.

[52] X. Zhang and Y. Gao, "Face recognition across pose: A review," Pattern Rec., vol. 42, no. 11, pp. 2876-2896, 2009.

[53] X. Zhang, Y. Gao, and T. Caelli, "Colour adjustment and specular removal for non-uniform shape from shading," in Proc. Australian Conf. Digital Image Computing: Techniques Applications, 2010, pp. 563-568.

[54] Q. Zheng and R. Chellappa, "Estimation of illuminant direction, albedo, and shape from shading," IEEE Trans. Pattern Anal. Mach. Intell., vol. 13, no. 7, pp. 680-702, Jul. 1991

[55] S. K. Zhou, G. Aggarwal, R. Chellappa, and D. W. Jacobs, "Appearance characterization of linear Lambertian objects, generalized photometric stereo, and illumination-invariant face recognition," IEEE Trans. Pattern Anal. Mach. Intell., vol. 29, no. 2, pp. 230-245, Feb. 2007.

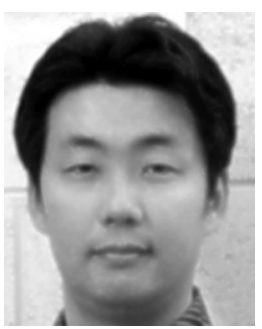

Xiaozheng Zhang received the B.Eng. degree in mechanical engineering from Tsinghua University, China, in 2001, and the Ph.D. degree in computer science from Griffith University, Australia, in 2008.

Currently, he is a Researcher with Queensland Research Laboratory, National ICT, Australia, and an Adjunct Research Fellow with the Institute for Integrated and Intelligent Systems, Griffith University, Australia. His research interests include computer vision, image processing, and pattern recognition. Particular interests are in the fields of face recognition, general 3-D modelling, and surface reflectivity for 3-D object rendering and reconstruction.

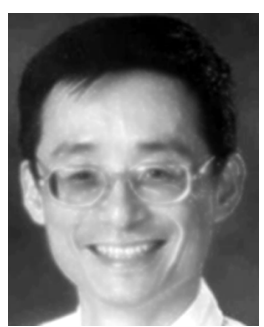

Yongsheng Gao (SM'02) received the B.Sc. and M.Sc. degrees in electronic engineering from Zhejiang University, China, in 1985 and 1988, respectively, and the $\mathrm{Ph} . \mathrm{D}$. degree in computer engineering from Nanyang Technological University, Singapore.

Currently, he is an Associate Professor with the Griffith School of Engineering, Griffith University, Australia. He is also with National ICT Australia, Queensland Research Laboratory, leading the Biosecurity group. His research interests include face recognition, biometrics, biosecurity, image retrieval, computer vision, and pattern recognition. 